\title{
Thermal Deformation Analysis Using Modelica
}

\author{
Eunkyeong Kim ${ }^{1} \quad$ Tatsurou Yashiki $^{1} \quad$ Fumiyuki Suzuki $^{2} \quad$ Yukinori Katagiri $^{1} \quad$ Takuya Yoshida $^{1}$ \\ ${ }^{1}$ Hitachi, Ltd., Research \& Development Group, Japan, \\ \{eunkyeong.kim.mn, tatsuro.yashiki.zn, yukinori.katagiri.gf, \\ takuya.yoshida.ru\}@hitachi.com \\ ${ }^{2}$ Mitsubishi Hitachi Power Systems, Ltd., Japan, \\ fumiyuki_suzuki@mhps.com
}

\begin{abstract}
This paper presents a thermal deformation analysis method fully utilizing the non-causality of the Modelica language as a means of solving large scale simultaneous equations including equilibrium equations related to stresses, stress-strain relations and strain-displacement relations. As an illustrative example, a model for thermal deformation analysis of a cylindrical object in the two-dimensional circular polar coordinate system is described. Simulations are performed for a cylindrically shaped object under a uniform temperature distribution and a radial temperature distribution. The results of the simulations show that the differences in displacements between the proposed model and a model based on finite element (FE) methods are less than $9 \%$ while the number of elements that compose the proposed model is about $1 / 8$ compared to that of the FE model.
\end{abstract}

Keywords: thermal deformation, stress-strain relation, strain-displacement relation, equilibrium equations, displacement gradient, physical modeling, finite volume method, non-causality, Modelica, Dymola

\section{Introduction}

Thermal deformation is conventionally analyzed by FE methods. Conventional FE methods supply highly precise outputs; however they require a lot of work and time. In order to conveniently analyze thermal deformation without using FE methods, analytical solutions have been extensively reported in the literature (see for example, Gere, 2006). These analytical solutions can only be used for simple geometries such as blocks and round bars and simple temperature distributions such as steady-state conditions. This is because it is difficult to analytically solve large scale simultaneous equations including equilibrium equations related to stresses, stress-strain relations and strain-displacement relations.

In this paper, a method to loosen these restrictions of simple geometries and simple temperature distributions is presented. The non-causality of the Modelica language (Elmqvist and Mattsson, 1997a, 1997b; Elmqvist et al, 1998a, 1998b; Fritzson and Engelson,
1998; Fritzson, 2003, 2011) is fully utilized as a means of formulating and solving the large scale simultaneous equations of thermal deformation analysis. The main feature of the proposed method is that the positions of displacements and forces are defined based on a finite volume method (Ferziger and Peric, 2002; Voller, 2009) to effectively describe these complex physical phenomena in non-causal manner and also to implement them as a network of Modelica component models.

\section{Thermal deformation model}

Here a model for the thermal deformation analysis of a cylindrical object in the two-dimensional circular polar coordinate system is described.

A cylindrical object represented by one quarter as illustrated in Figure 1 is discretized the 9 control volumes (referred to as elements in this paper) by dividing the object in the $r$ and $\theta$ directions into $3 \times 3$ elements. A graphical image of the Modelica component models interacting with each other for analyzing thermal deformation of the cylindrical object is shown in Figure 2. There are 3 types of component models; a model for calculating the force balances inside the element (element model), a model for calculating the interactions of forces and deformations between elements (linkage model), and a model for calculating the boundary conditions (BC model). There are also connectors designed to connect these component models so that structural analysis of the entire object is performed (SA connector).

In the following, the SA connector (Sec. 2.1), the element model (Sec. 2.2), the linkage model (Sec. 2.3) and the BC model (Sec. 2.4) are described, and then the method to calculate the displacements at the vertices of the elements (Sec. 2.5) is given. 


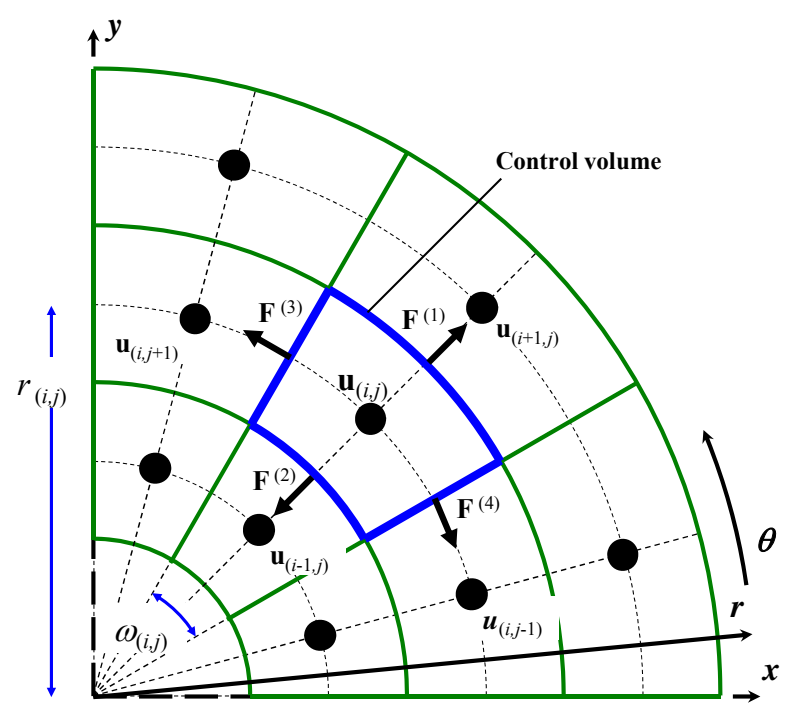

Figure 1. A cylindrical object and its discretization.

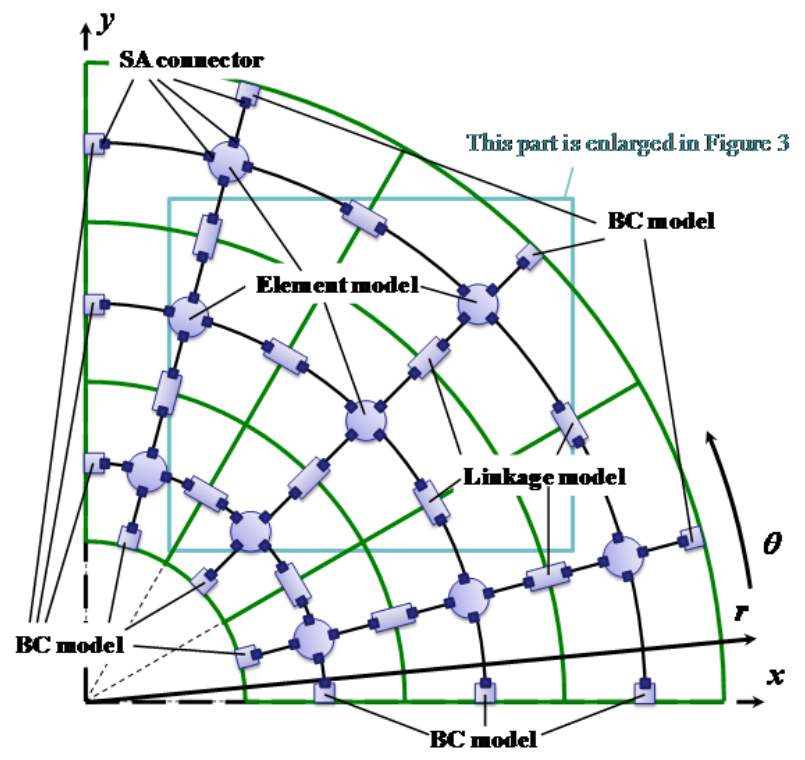

Figure 2. A graphical image of the Modelica component models for analyzing thermal deformations of the cylindrical object in Figure 1.

Table 1. Declarations of variables in the SA connector

\begin{tabular}{|l|l|l|}
\hline \multicolumn{1}{|c|}{ Type } & Name & \multicolumn{1}{c|}{ Description } \\
\hline Length & ur & displacement in the $r$ direction \\
\hline Length & ut & displacement in the $\theta$ direction \\
\hline Real & durdr & displacement gradient $\partial u_{r} / \partial r$ \\
\hline Real & dutdr & displacement gradient $\partial u_{\theta} / \partial r$ \\
\hline Real & durdt & displacement gradient $\partial u_{r} / \partial \theta$ \\
\hline Real & dutdt & displacement gradient $\partial u_{\theta} / \partial \theta$ \\
\hline flow Force & Fr & force acting in the $r$ direction \\
\hline flow Force & Ft & force acting in the $\theta$ direction \\
\hline
\end{tabular}

\subsection{SA connector}

The SA connector passes the information of forces, displacements, and displacement gradients between the component models. Each SA connector has 6 variables as shown in Table 1: 2 variables representing displacements at the center position of the element; 4 variables representing displacement gradients; and 2 variables representing forces acting on a boundary surface of the element which are the flow variables.

\subsection{Element model}

In this model, (1) the balance of the forces acting on the boundary surfaces of an element is calculated, and also (2) the displacement gradients at the center position of the element are calculated and passed to the SA connectors, then (3) the information of the displacements at the center position is passed to the SA connectors.

Each element model has 6 dynamic variables as shown in Table 2: 2 variables representing displacements at the center position of the element; and 4 variables representing displacements gradients at the center position of the element. The information of displacements and displacement gradients are passed to the linkage models located on the boundary surfaces of the element via the $4 \mathrm{SA}$ connectors Crb, Cra, Ctb and Cta for interacting with neighbor elements (The connections between the element model and the linkage models via the SA connectors are shown in Figure 3).

(1) The balance of the forces acting on the boundary surfaces of an element is calculated by

$\mathbf{F}^{(1)}+\mathbf{F}^{(2)}+\mathbf{F}^{(3)}+\mathbf{F}^{(4)}=\mathbf{0}$

where $\mathbf{F}^{(1)}, \mathbf{F}^{(2)}, \mathbf{F}^{(3)}$ and $\mathbf{F}^{(4)}$ are representing the forces acting on boundary surfaces 1, 2, 3 and 4 (Figure 1). This equation is described by the following Modelica code.

Crb.Fr+Cra.Fr+Ctb.Fr+Cta.Fr=0 ;

$\mathrm{Crb} \cdot \mathrm{Ft}+\mathrm{Cra} \cdot \mathrm{Ft}+\mathrm{Ctb} \cdot \mathrm{Ft}+\mathrm{Cta} \cdot \mathrm{Ft}=0$;

(2) The displacement gradients at the center position of the element are calculated by

$$
\begin{aligned}
& \left(\frac{\partial u_{\xi}}{\partial r}\right) \approx \frac{1}{2}\left[\left(\frac{\partial u_{\xi}}{\partial r}\right)^{(1)}+\left(\frac{\partial u_{\xi}}{\partial r}\right)^{(2)}\right] \\
& \left(\frac{\partial u_{\xi}}{\partial \theta}\right) \approx \frac{1}{2}\left[\left(\frac{\partial u_{\xi}}{\partial \theta}\right)^{(3)}+\left(\frac{\partial u_{\xi}}{\partial \theta}\right)^{(4)}\right]
\end{aligned}
$$

where $u_{\xi}$ denotes the displacements $u_{r}$ and $u_{\theta}$. The displacement gradients in the $r$ direction $\left(\partial u_{\xi} / \partial r\right)$ are calculated as the averages of $\left(\partial u_{\xi} / \partial r\right)$ on boundary surfaces 1 and 2. The displacement gradients in the $\theta$ direction $\left(\partial u_{\xi} / \partial \theta\right)$ are calculated as the averages of 
$\left(\partial u_{\xi} / \partial \theta\right)$ on boundary surfaces 3 and 4 . These equations are described by the following Modelica code.

durdr $=(\mathrm{Crb} \cdot \mathrm{durdr}+\mathrm{Cra} \cdot \mathrm{durdr}) / 2$;

dutdr $=(\mathrm{Crb} \cdot$ dutdr+Cra $\cdot$ dutdr $) / 2$;

durdt $=(C t b \cdot d u r d t+C t a \cdot d u r d t) / 2$;

dutdt $=(\mathrm{Ctb} \cdot d u t d t+\mathrm{Cta} \cdot$ dutdt $) / 2$;

The displacement gradients with respect to $r$ or $\theta$ axis are passed to the linkage models located on the boundary surfaces along the other axis via the SA connectors. The Modelica code to form these information flows is as follows.

Ctb. durdr=durdr:

ctb.dutdr=dutdr;

Cta.durdr=durdr;

Cta.dutdr=dutdr;

Crb.durdt=durdt ;

Crb.dutdt $=$ dutdt ;

Cra.durdt =durdt ;

Cra.dutdt $=$ dutdt;

(3) The displacements at the center position of the element are determined by the interactions of forces and displacements between the neighbor elements calculated by the linkage models mentioned in section 2.3. The Modelica code to share the information of the displacements among the surrounding linkage models is as follows.

Crb.ur=ur;

Cra . ur=ur;

Ctb.ur=ur;

Cta.ur=ur;

Crb.ut $=u t$;

Cra.ut $=u t$;

Ctb.ut $=u t$;

cta.ut $=u t$;

Table 2. Declarations of variables in the element model

\begin{tabular}{|l|l|l|}
\hline \multicolumn{1}{|c|}{ Type } & \multicolumn{1}{c|}{ Name } & \multicolumn{1}{c|}{ Description } \\
\hline Length & ur & displacement in the $r$ direction \\
\hline Length & ut & displacement in the $\theta$ direction \\
\hline Real & durdr & displacement gradient $\partial u_{r} / \partial r$ \\
\hline Real & dutdr & displacement gradient $\partial u_{\theta} \partial r$ \\
\hline Real & durdt & displacement gradient $\partial u_{r} / \partial \theta$ \\
\hline Real & dutdt & displacement gradient $\partial u_{\theta} \partial \theta$ \\
\hline
\end{tabular}

Table 3. Declarations of variables in the linkage model and the $\mathrm{BC}$ model

\begin{tabular}{|l|l|l|}
\hline \multicolumn{1}{|c|}{ Type } & \multicolumn{1}{|c|}{ Name } & \multicolumn{1}{c|}{ Description } \\
\hline Stress & tau rr & stress in the $r$ direction \\
\hline Stress & tau rt & stress in the $\theta$ direction \\
\hline Real & epsilon $r r$ & strain in the $r$ direction \\
\hline Real & epsilon $r t$ & shear strain \\
\hline Real & epsilon tt & strain in the $\theta$ direction \\
\hline Real & durdr & displacement gradient $\partial u_{r} / \partial r$ \\
\hline Real & dutdr & displacement gradient $\partial u_{\theta} \partial r$ \\
\hline Real & durdt & displacement gradient $\partial u_{r} / \partial \theta$ \\
\hline Real & dutdt & displacement gradient $\partial u_{\theta} \partial \theta$ \\
\hline
\end{tabular}

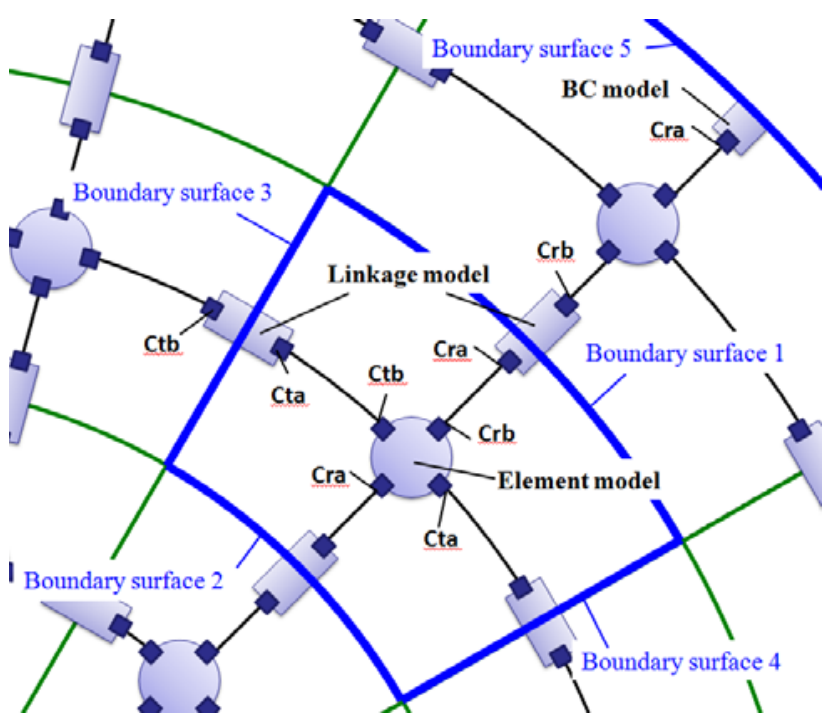

Figure 3. Enlarged illustration of Figure 2.

\subsection{Linkage model}

In this model, (1) the forces acting on the boundary surfaces, (2) the stress-strain relations, (3) the straindisplacement relations, and (4) the displacement gradients on the boundary surfaces between the elements are calculated, and then the resulting displacement gradients are passed to the SA connectors.

There are several types of linkage model according to the number of coordinates: in this paper, the first is for dealing with the relations between the adjacent elements in the $r$ direction and the second is for dealing with those in the $\theta$ direction. Here the model concerning boundary surface 1 located in the $r$ direction (Figure 3 ) is described as an example.

The linkage model has 9 dynamic variables as shown in Table 3: 2 variables representing stresses acting on boundary surface $1 ; 3$ variables representing strains on boundary surface 1 ; and 4 variables representing displacement gradients on boundary surface 1. This information on displacement gradients is passed to the element models adjacent to the linkage model via the 2 SA connectors Crb and Cra.

(1) The force acting on boundary surface 1 is calculated by

$\mathbf{F}^{(1)}=\left[\begin{array}{c}\tau_{r r}{ }^{(1)} \\ \tau_{r \theta}{ }^{(1)}\end{array}\right] r^{(1)} \omega L_{z}$

This equation is described by the following Modelica code.

Crb. Fr =tau rr*r*omega* Lz ;

$\mathrm{Crb} . \mathrm{Ft}=$ tau rt*r*omega* Lz;

Cra.Fr=-Crob.Fr;

Cra.Ft $=-$ Crb.Ft;

where $r$ is the radius at this boundary surface, omega is the angle of the element, and $\mathrm{Lz}$ is the axial length of the element. 
(2) The stress-strain relations on boundary surface 1 are calculated by the following equations.

$$
\begin{aligned}
\tau_{r r}{ }^{(1)} & =\frac{E}{1-v^{2}}\left[\varepsilon_{r r}{ }^{(1)}+v \varepsilon_{\theta \theta}{ }^{(1)}-\alpha(1+v)\left(T^{(1)}-T_{0}\right)\right] \\
\tau_{r r}{ }^{(1)} & =\frac{E}{(1+v)(1-2 v)} \\
& {\left[(1-v) \varepsilon_{r r}{ }^{(1)}+v \varepsilon_{\theta \theta}{ }^{(1)}-\alpha(1+v)\left(T^{(1)}+T_{0}\right)\right] } \\
\tau_{r \theta}{ }^{(1)} & =\frac{E}{2(1+v)} \varepsilon_{r \theta}{ }^{(1)}
\end{aligned}
$$

Equation (5) is used for the plane stress problem in which the whole vertical stress on the $r-\theta$ plane is zero, and equation (6) is used for the plane strain problem in which the whole vertical strain on the $r-\theta$ plane is zero (Voller, 2009). For example, the Modelica code to deal with the plane stress problem is as follows.

tau rr $=\mathrm{E} /\left(1-\mathrm{nu}^{\wedge} 2\right)$ * (epsilon_rr+

nu*epsilon tt $-(1+\mathrm{nu}) * a l$ pha $\overline{\text { ep }}(\mathrm{Temp}-\mathrm{Temp} 0))$. tau_rt $=E / 2 \bar{T}(1+n u) *$ epsilon_rt;

where E is Young's modulus, nu is the Poisson ratio, alpha is the coefficient of thermal expansion, Temp 0 is the initial temperature, and Temp is the temperature.

(3) The strain-displacement relations on the boundary surface 1 are calculated by

$\varepsilon_{r r}^{(1)}=\left(\frac{\partial u_{r}}{\partial r}\right)^{(1)}$

$\varepsilon_{\theta \theta}^{(1)}=\frac{u_{r}^{(1)}}{r^{(1)}}+\frac{1}{r^{(1)}}\left(\frac{\partial u_{\theta}}{\partial \theta}\right)^{(1)}$

$\varepsilon_{r \theta}^{(1)}=\frac{1}{r^{(1)}}\left(\frac{\partial u_{r}}{\partial \theta}\right)^{(1)}+\left(\frac{\partial u_{\theta}}{\partial r}\right)^{(1)}-\frac{u_{\theta}{ }^{(1)}}{r^{(1)}}$

where $u_{r}{ }^{(1)}$ and $u_{\theta}{ }^{(1)}$ denote the displacements on boundary surface 1 and they are approximated by the averages of the displacements of the adjacent elements.

$u_{\xi}^{(1)} \approx \frac{u_{\xi(i+1, j)}+u_{\xi(i, j)}}{2}$

Here $u_{\xi}$ means the displacements $u_{r}$ and $u_{\theta}$. These relations are described by the following Modelica code. epsilon rr=durdr;

epsilon_tt $=(\mathrm{Crb} \cdot \mathrm{ur}+\mathrm{Cra} \cdot \mathrm{ur}) / 2 / \mathrm{r}+\mathrm{dutdt} / \mathrm{r}$; epsilon rt=durdt/r+dutdr-

(Crb.ut+Cra.ut) /2/r;

(4) The displacement gradients perpendicular to boundary surface 1 are approximated from the displacements of the adjacent elements.

$\left(\frac{\partial u_{\xi}}{\partial r}\right)^{(1)} \approx \frac{u_{\xi(i+1, j)}-u_{\xi(i, j)}}{r_{(i+1, j)}-r_{(i, j)}}$

This equation is described by the following Modelica code.

durdr $=($ Crb $\cdot u r-C r a \cdot u r) /(r b-r a)$;

dutdr $=($ Crb.ut-Cra.ut $) /\left(r^{-} b-r^{-} a\right)$;

where $r_{-} b$ and $r_{-} a$ are the radii at the center positions

of the adjacent elements.
The displacement gradients are passed to the element models adjacent to boundary surface 1 via the SA connectors. The Modelica code to form these information flows is as follows.

$\mathrm{Crb}$.durdr=durdr;

Cra. durdr=durdr;

Crb.dutdr=dutdr;

Cra.dutdr=dutdr;

The displacement gradients tangential to boundary surface 1 are interpolated using the values at the center positions of the adjacent elements.

$\left(\frac{\partial u_{\xi}}{\partial \theta}\right)^{(1)} \approx \frac{1}{2}\left[\left(\frac{\partial u_{\xi}}{\partial \theta}\right)_{(i+1, j)}+\left(\frac{\partial u_{\xi}}{\partial \theta}\right)_{(i, j)}\right]$

This equation is described by the following Modelica code.

durdt $=($ Crb.durdt + Cra.durdt $) / 2$;

dutdt $=($ Crb.dutdt + Cra.$d u t d t) / 2$;

\subsection{BC model}

This model is located on the boundaries of the entire object (Figure 2). The $\mathrm{BC}$ model is similar to the linkage model; however it is different in that it deals with either a restraint or a loading condition that has to set the boundaries of the entire object.

Here the model concerning boundary surface 5 located in the $r$ direction (Figure 3 ) is described as an example.

The $\mathrm{BC}$ model has 9 dynamic variables as shown in Table 3, and the information of displacement gradients is passed to the element model adjacent to boundary surface 5 via the SA connector Cra.

(1) The restraint condition is represented using the displacement gradients.

$\left(\frac{\partial u_{\xi}}{\partial r}\right)^{(5)} \approx \frac{u_{\xi}{ }^{\mathrm{BC}}-u_{\xi(i+1, j)}}{r^{\mathrm{BC}}-r_{(i+1, j)}}$

$\left(\frac{\partial u_{\xi}}{\partial \theta}\right)^{(5)} \approx\left(\frac{\partial u_{\xi}}{\partial \theta}\right)_{(i+1, j)}$

Here $u_{\xi}$ denotes the displacements $u_{r}$ and $u_{\theta}, u_{\zeta}^{\mathrm{BC}}$ is the displacements of boundary surface 5 , and $r^{\mathrm{BC}}$ is the radius on boundary surface 5 . These equations are described by the following Modelica code.

durdr $=\left(u r \_b c-C r a . u r\right) /\left(r \_b c-r\right)$; dutdr $=\left(u^{-} \mathrm{bc}-\mathrm{Cra} \cdot \mathrm{ut}\right) /\left(\mathrm{r}_{\mathrm{b}} \mathrm{b}-\mathrm{r}\right)$; durdt $=$ Cra. durdt ;

dutdt=Cra. dutdt; 
(2) The loading condition is represented by

$\mathbf{F}^{\mathrm{BC}} \approx\left[\begin{array}{c}\tau_{r r}{ }^{(5)} \\ \tau_{r \theta}{ }^{(5)}\end{array}\right] r^{\mathrm{BC}} \omega L$

$\left(\frac{\partial u_{\xi}}{\partial \theta}\right)^{(5)} \approx\left(\frac{\partial u_{\xi}}{\partial \theta}\right)_{(i+1, j)}$

where $\mathbf{F}^{\mathrm{BC}}$ is the force acting on boundary surface 5 . This equation is described by the following Modelica code.

$\mathrm{Fr}$ bc=tau_rr*r_bc*omega*Lz;

$\mathrm{Ft}-\mathrm{bc}=\mathrm{tau}^{-} \mathrm{rq}^{*} \mathrm{r}^{-} \mathrm{bc} \mathrm{c}^{*}$ omega ${ }^{-} \mathrm{Lz}$;

dur $d t=$ Cra.. durd $\bar{t}$;

dutdt $=$ Cra. dutdt;

\subsection{Calculation of the displacement at the vertices of the elements}

In this section, a method for calculating the displacements at the vertices of the elements $(\bigcirc$ marks in Figure 4) is described using an example illustrated in Figure 4. Here the displacements at the center positions $(\diamond$ marks in Figure 4 ) have already been obtained by the models described in the previous sections.

The displacements at the vertices are calculated using the positons, the displacements and the displacement gradients of the surrounding elements. First, the displacement of the position $\mathbf{r}_{1}$ is obtained using the information of the position $\mathbf{r}_{2}$ as follows.

$\mathbf{u}_{1 f\left(\mathbf{r}_{2}\right)}=\mathbf{u}_{2}+\operatorname{grad}\left(\mathbf{u}_{2}\right)\left(\mathbf{r}_{1}-\mathbf{r}_{2}\right)$

Also the same displacement of the position $\mathbf{r}_{1}$ is obtained using the information of the other positions $\mathbf{r}_{3}$, $\mathbf{r}_{4}$, and $\mathbf{r}_{5}$, in the same manner. The displacement of this point is defined as the average of these values obtained using the information of the surrounding elements.

$\mathbf{u}_{1}=\frac{1}{4}\left(\mathbf{u}_{1 f\left(\mathbf{r}_{2}\right)}+\mathbf{u}_{1 f\left(\mathbf{r}_{3}\right)}+\mathbf{u}_{1 f\left(\mathbf{r}_{4}\right)}+\mathbf{u}_{1 f\left(\mathbf{r}_{5}\right)}\right)$

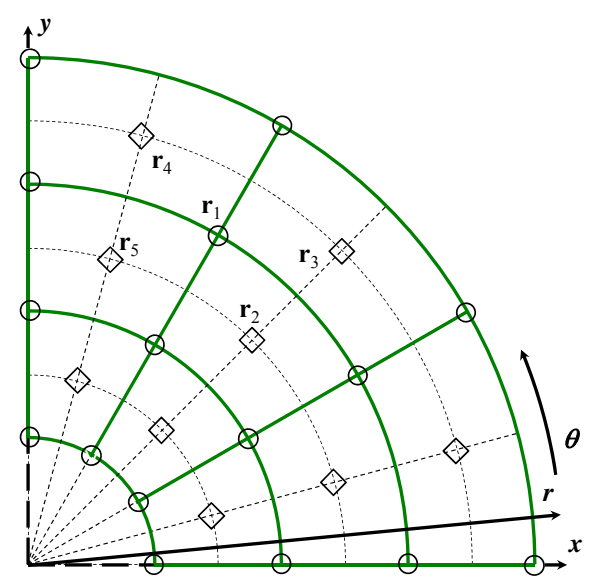

Figure 4. Vertices and center positions of the elements.

\section{Simulation examples}

The simulations were performed for a cylindrically shaped object using Dymola and the results were compared with those obtained by FE analyses.

\subsection{Simulation target and conditions}

Figure 5 shows the simulation target and examples of its FE analyses. The object consisted of the separate upper and lower halves of cylindrically shaped parts, and the upper half was placed on the lower half. Since this object was symmetrical between the left and right sides, the left half was analyzed. The analyses were performed for two cases of temperature conditions: a uniform temperature under which the object was thermally expanded in a uniform manner (Figure 5 (a), Case 1), and a radial temperature distribution under which both the upper and lower halves were deformed (Figure 5 (b), Case 2). To deal with the original three dimensional deformation by the two dimensional model, the deformation was analyzed in several $r-\theta$ planes on which the plane stress problem was applied.

Figure 6 (a) shows the discretization of the simulation target for the proposed method. A quarter was analyzed assuming that the upper and lower halves having the same geometries deformed symmetrically. The original shape (not exactly a cylinder) used in the above FE analyses was simplified into an exact quarter cylinder; the inner and outer radii were determined so that the volume was kept equal to the original. The object was discretized into 60 elements $(4 \times 15$ in the $r$ and $\theta$ directions). For the boundary conditions, the circumferential displacements on one side of the circumferential boundary surfaces were restrained. The length $L_{y}$ in the $y$ direction between the positions $\mathrm{A}$ and $B$ was used to evaluate the accuracy of the proposed method.

Figure 6 (b) shows the temperature conditions for Case 1 and 2 . In Case 1, the object was maintained at a uniform temperature of $486^{\circ} \mathrm{C}$. In Case 2, the inner surface of the object was maintained at $486^{\circ} \mathrm{C}$ and the outer surface at $342^{\circ} \mathrm{C}$. The initial temperature before the occurrences of the thermal deformations was determined to be $15^{\circ} \mathrm{C}$. 


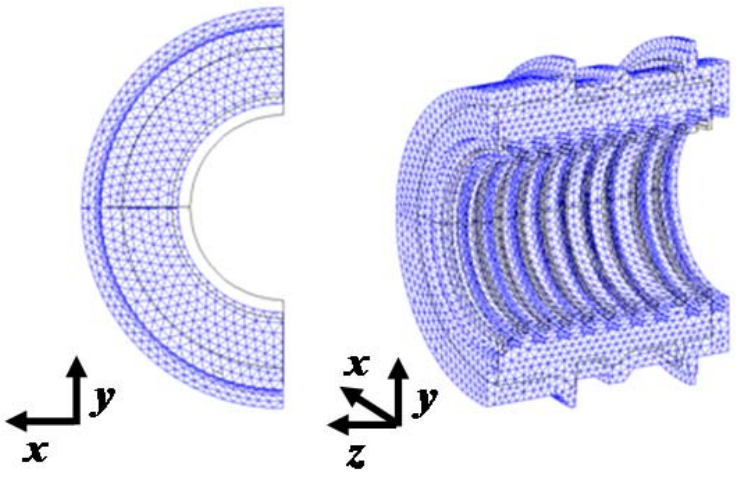

(a) A uniform temperature distribution (Case 1)
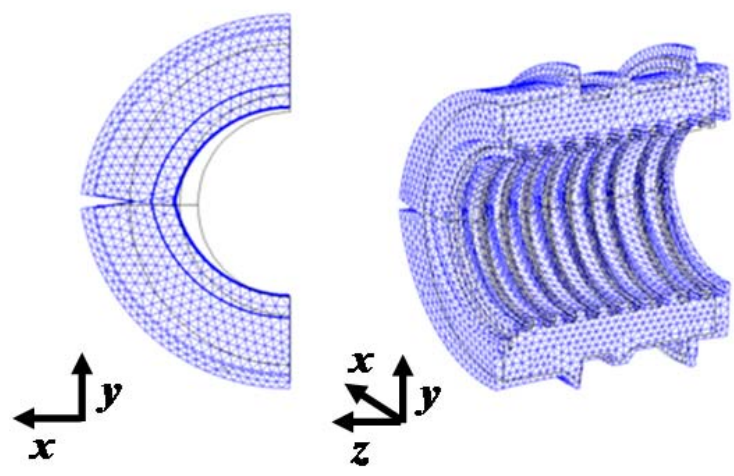

(b) A radial temperature distribution (Case 2)

Figure 5. Simulation target.

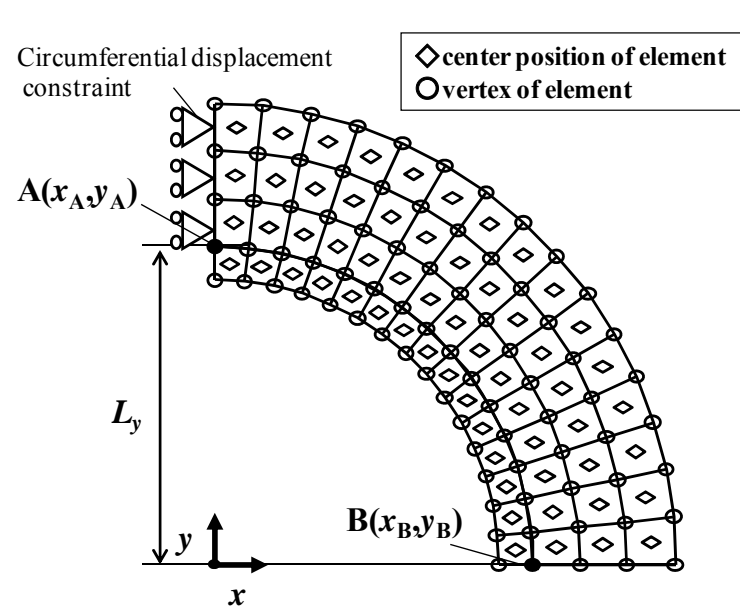

(a) Discretization of the simulation target for evaluating the proposed model

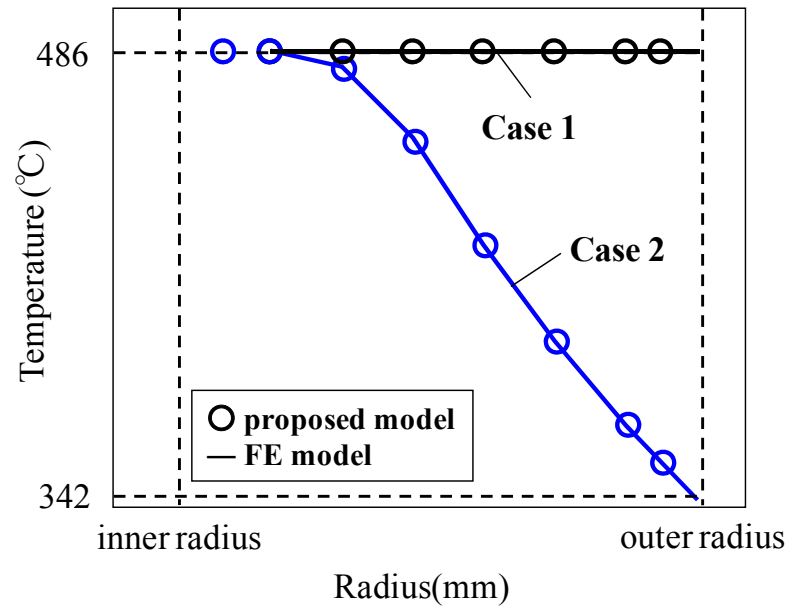

(b) Temperature conditions

Figure 6. Simulation conditions.
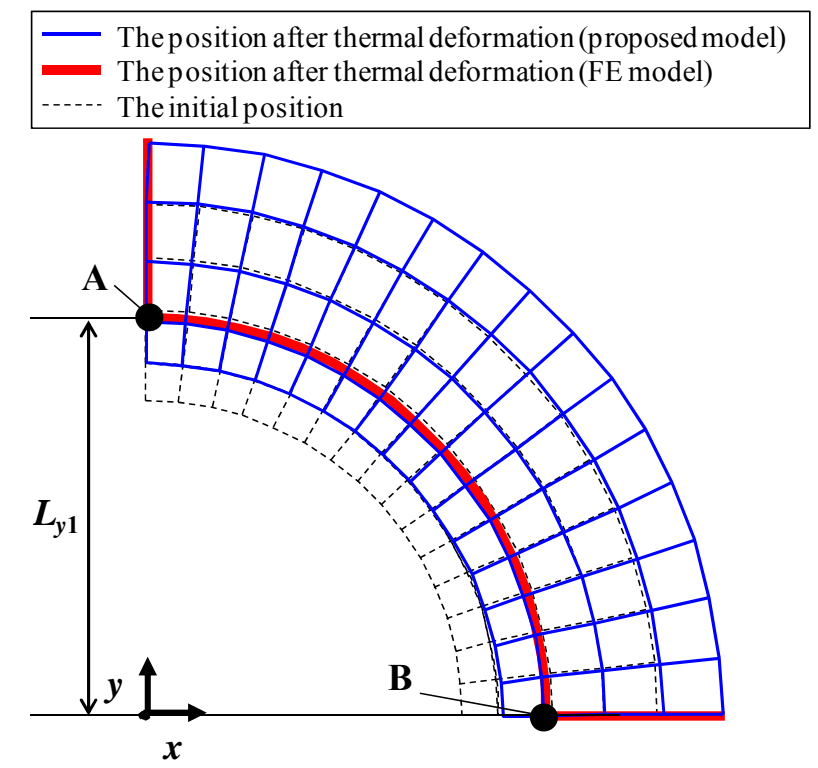

(a) A uniform temperature distribution (Case 1)
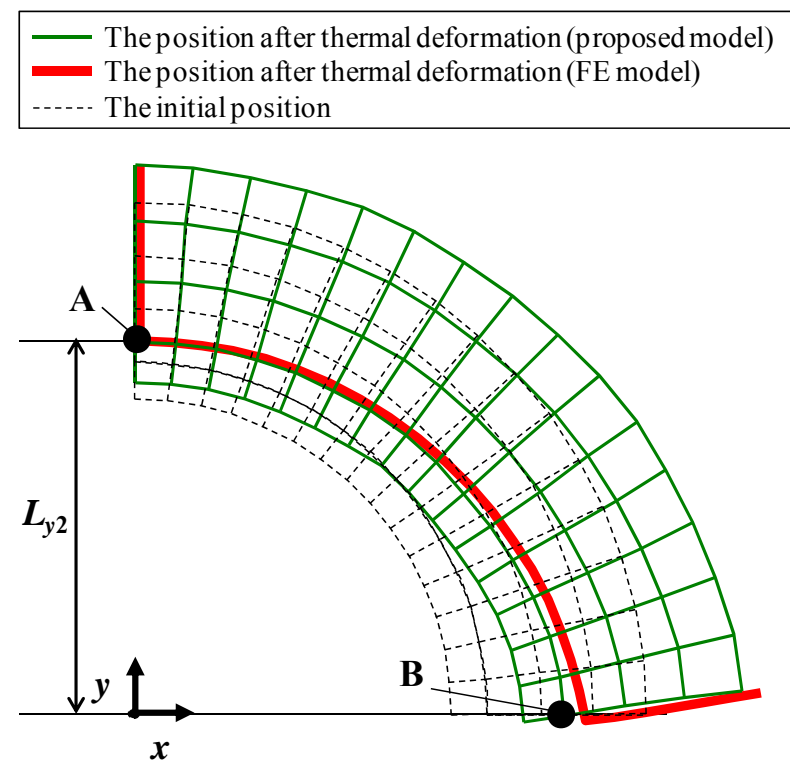

(b) A radial temperature distribution (Case 2)

Figure 7. Simulation results. The displacements were magnified 20 times. 
Table 4. Differences in the number of elements and the simulation results between the proposed model and the FE model.

\begin{tabular}{|l|r|r|r|}
\hline & Proposed model & FE model & Difference \\
\hline Number of elements in the $x-y$ plane & 60 & 500 & - \\
\hline Length of initial condition $\left(L_{y 0}\right)$ & $1(-)^{* 1}$ & $1(-)^{* 1}$ & - \\
\hline Displacment of Case 1 $\left(\Delta L_{y 1}=L_{y 1}-L_{y 0}\right)$ & $6.0 \times 10^{-3}(-)^{* 1}$ & $6.5 \times 10^{-3}(-)^{* 1}$ & $-7.6(\%)^{* 2}$ \\
\hline Displacment of Case 2 $\left(\Delta L_{y 2}=L_{y 2}-L_{y 0}\right)$ & $3.1 \times 10^{-3}(-)^{*}{ }^{* 1}$ & $3.7 \times 10^{-3}(-)^{* 1}$ & $-9.0(\%)^{* 2}$ \\
\hline
\end{tabular}

*1: Dimensionless value using the length of the initial condition $L_{y 0}$

*2: Difference $(\%)=\left(\Delta L_{y_{-} \text {Proposed }}-\Delta L_{y_{-} \mathrm{FE}}\right) / \Delta L_{y_{-} \mathrm{FE}} \times 100$

\subsection{Simulation results}

Figure 7 (a) and (b) show the initial positon and the positions after thermal deformations in Case 1 and 2, respectively. The positions after thermal deformations were compared those calculated by an FE model. The number of elements in the $x-y$ plane of the FE model was about 500 (The total number of elements in three dimensions was 26335). The simulation results obtained by the proposed model were in good agreement with those obtained by the FE model in both Cases 1 and 2.

The differences in the number of elements and the simulation results between the proposed model and the FE model in both Cases 1 and 2 are summarized in Table 4. The differences in simulated displacements were less than $9 \%$ while the number of elements that compose the proposed model was about $1 / 8(\approx 60 / 500)$ compared to that of the FE model.

\section{Summary and discussion}

A thermal deformation analysis method was proposed that fully utilizes the non-causality of the Modelica language as a means of formulating and solving large scale simultaneous equations including equilibrium equations related to stresses, stress-strain relations and strain-displacement relations. The main feature of the proposed method is that the positions of displacements and forces are defined based on a finite volume method to effectively describe these complex physical phenomena in a non-causal manner and also to implement them as a network of Modelica component models.

As an illustrative example, a Modelica model for thermal deformation analysis of a cylindrical object in a two-dimensional circular polar coordinate system was presented. Simulations were performed for a cylindrically shaped object under a uniform temperature distribution and a radial temperature distribution. The results of simulations showed that the differences in the displacements between the proposed model and the FE model were less than $9 \%$ while the number of elements that compose the proposed model was about $1 / 8$ compared to that of the FE model.
The proposed method can deal with more complex thermal deformation analyses than presently used analytical solutions and can obtain precise outputs comparable to that of FE methods with a fewer number of elements. These advantages make it possible to perform thermal deformation analyses in system level Modelica simulations containing structural objects and control devices. Furthermore, the method should be applicable to optimizations of geometries of such objects or control systems.

\section{Nomenclature}

A position

$B$ position

E Young's modulus

F force vector

$G \quad$ shear modulus $(=E / 2 /(1+v))$

$L_{y} \quad$ length between positions A and B

$L_{y 0} \quad$ length of $L_{y}$ in the initial condition

$L_{y 1} \quad$ length of $L_{y}$ in case 1

[m]

$L_{y 2} \quad$ length of $L_{y}$ in case 2

$L_{z} \quad$ axial length of an element

[m]

r position vector

[m]

[m]

$r \quad$ radius

$T_{0} \quad$ temperature at the initial condition

$u_{r} \quad$ displacement in the radial direction direction coefficient of linear expansion strain in the radial direction shear strain strain in the circumferential direction

$\tau_{r r} \quad$ stress in the radial direction

$\tau_{r \theta} \quad$ shear stress 


\section{Subcripts}

$f(\mathbf{r}) \quad$ calculated using the positions, displacements and displacement gradients of position $\mathbf{r}$

$i \quad$ number of elements in the radial direction

$j \quad$ number of elements in the circumferential direction

$\xi \quad$ in the radial and circumferential directions

Supercripts

BC boundary surface of simulation target

(1)-(4) boundary surface of element

(5) between boundary surface 5 and center position

\section{References}

Elmqvist H., Mattsson S.E. (1997a): Modelica - The next generation modeling language an international design effort, Proceedings of the 1st World Congress on System Simulation

Elmqvist H., Mattsson S.E. (1997b): An introduction to the physical modeling language Modelica, Proceedings of the 9th European Simulation Symposium

Elmqvist H, Mattsson S.E., Otter M. (1998a): Modelica The new object-oriented modeling language, 12th European Simulation Multiconference

Elmqvist H., Mattsson S.E., Otter M. (1998b): Modelica An international effort to design an object-oriented modeling language, Summer Computer Simulation Conference

Ferziger J.H., Peric M. (2002): Computational Methods for Fluid Dynamics, Springer-Verlag, pp.21-37

Fritzson P. (2003): Principles of object-oriented Modeling and Simulation with Modelica 2.1, John Wiley \& Sons, Inc., pp.19-25

Fritzson P. (2011): Introduction to Modeling and Simulation of Technical and Physical Systems with Modelica, John Wiley \& Sons, Inc., pp.29-39

Fritzson P., Engelson V. (1998): Modelica-A unified object-oriented language for system modeling and simulation, ECOOP'98, LNCS 1445, pp. 67-90

Gere J.M. (2006), Mechanics of meterials, Thomson learning, pp.93-104

Voller V.R. (2009): Basic control volume finite element methods for fluids and solids, World Scientific Publishing Co. Pte. Ltd., pp.10-20 